
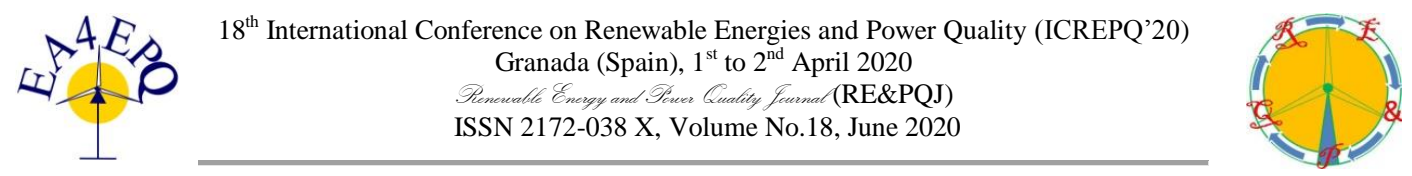

\title{
Analysis of resonance modes at harmonic frequencies in high-voltage networks
}

\author{
L.I. Kovernikova \\ The Siberia Branch of the Russian Academy of Sciences \\ Melentiev Energy Systems Institute \\ 130, Lermontov Str., 664033, Irkutsk (Russia) \\ Phone: +7 3952500646 ext. 232, fax: +7 3952 426796, e-mail: kovernikova@ isem.irk.ru
}

\begin{abstract}
Resonant modes in electric networks occur due to the presence of capacitive and inductive elements which compensate each other. Resonant circuits can occur on harmonic frequencies (further referred to as harmonics). If the networks have consumers of electric power having equipment with nonlinear current-voltage characteristics that consumes a nonsinusoidal current, then the harmonics of currents and voltages in the resonant circuits sharply increase. Harmonics create energy losses in electrical equipment, which causes its additional heating, leads to a reduction in service life and, as a result, additional economic damage. Passive filters and capacitors, which are used to compensate reactive power, are especially vulnerable in such cases.
\end{abstract}

Resonant nodes of electrical network and resonant harmonics can be determined by calculating the frequency responses of the input impedances or admittances of the nodes for various network configurations and loads. The experience of studying harmonic modes in electric networks allowed us to develop a methodical approach and HARMONICS software for determining resonant nodes and resonant harmonics.

The article provides methodical approach and some results of the research on resonant modes on the harmonics in the high-voltage networks in Eastern Siberia.

Key words. Harmonics, resonance, frequency response, high-voltage network.

\section{Introduction}

Resonance modes at harmonic frequencies in electrical networks are a big problem [1]-[9]. For many years of power systems operation the regulatory documents keep pointing to the need of taking into account resonant modes on harmonics. The authors [10] indicate that "the nonsinusoidal voltage in the point of common coupling can be created both by distorting electrical receivers of consumers and the equipment of power supplying organizations operating the modes which contribute the manifestation of non-sinusoidal properties due to their current-voltage characteristics or the appearance of resonant modes". Despite the problem of resonant modes on harmonics and the attempts of its solution during a long time, it keeps being relevant. The authors of [11] suggest, when choosing reactive power compensation devices containing capacitor banks, to test them for possible overload by harmonic currents. "It is especially noted that the use of a capacitor bank is possible provided that resonance phenomena are excluded for all modes of operation of the electric network".

Resonant modes in electric networks occur due to the presence of capacitive and inductive elements which compensate each other on some harmonics [12]-[13]. In resonance modes, the harmonics of the current and voltage can increase to dangerous ones. Resonant modes or close to them can occur in one and the same network node on various harmonics. The voltage harmonics in resonant modes quite frequently exceed the limit values set by the state standard [14]. As a result of the appearance of large values of current and voltage harmonics damage to electrical equipment is possible [15]-[17]. Resonant circuits occur in a random way at the network changes caused by switching on/switching off the power lines, capacitors, filters, change in loads, etc., which causes additional problems at setting the causes of significant values of voltage and current harmonics in the network.

The article presents a methodical approach, the results of calculations with the HARMONICS software [18], which can be used in the operation of electric networks in order to predict resonance modes for controlling the power quality. It is very important to predict resonant modes as harmonic modes are random and mode parameters have stochastic nature [19]. The article provides some results of the research on resonant modes on the harmonics in the high-voltage networks in Eastern Siberia. 


\section{Methodical Approach for Analysing Resonant Modes on Harmonics}

Resonant modes on various harmonics occur in the electric network due to the presence of capacitive elements: capacitors used for compensating reactive power, passive filters, and capacities of cable and overhead power lines.

Two types of resonances can occur in the network: series (voltage resonance) and parallel (current resonance). Inductive reactance and capacitive reactance at series resonance are connected in series. On some harmonics their values become equal and they compensate each other. In this case, a large current can occur in the common circuit, and a large voltage can occur at the capacitive reactance and inductive reactance. At parallel resonance inductive reactance and capacitive reactance are connected in parallel. Their values become equal at some harmonic. Large value currents can flow through inductive reactance and capacitive reactance. Large value voltages can appear on each of the reactance of the resonant circuit. Types of resonances alternate in the nodes of the real electrical networks [12]-[13].

Network nodes in which resonances and resonant harmonics are possible can be determined by calculating the frequency responses of the input impedances or admittances of the network nodes for various circuit configurations and load powers. This is a difficult problem for the network of large dimension, because it is necessary to take into account the large number of network elements. The experience of researching harmonic modes allowed the author to develop the methodical approach and the HARMONICS software for practical calculations in order to predict resonant nodes and resonant harmonics. The content of the methodical approach is presented below.

Voltage harmonics in the network nodes can be calculated as a result of solving the equation system [20]

$$
\boldsymbol{U}_{\boldsymbol{h}}=\boldsymbol{Z}_{\boldsymbol{h}} \boldsymbol{I}_{\boldsymbol{h}},
$$

where $h$ - harmonic order; $\boldsymbol{U}_{\boldsymbol{h}}$ - column-matrix of nodal voltage values to be determined; $\boldsymbol{I}_{\boldsymbol{h}}$ - column-matrix of harmonic currents at network nodes representing load; $\boldsymbol{Z}_{\boldsymbol{h}}$

- matrix of self- and mutual impedances of the network nodes, which is obtained as result of inversion of the nodal admittance matrix $\boldsymbol{Y}_{\boldsymbol{h}}[20]$.

The parameters, used in the equation system (1), are applied for analyzing the network on the presence of resonances in the $i$-th network node with the help of the following parameters: $K_{U(h)}$ - the $h$-th harmonic factor; $K_{L^{-}}$limit values for $K_{U(h)}$ established in [14]; $I_{h, i}=\sum_{k=1}^{K} I_{h, k i}$ - current value of the $h$-th harmonics in the $i$-th node where $I_{h, k i}$ - the $h$-th harmonic current generated by the load with non-linear volt-ampere characteristics from the $\kappa$-th node to the $i$-th node; $y_{h, i}=1 / z_{h, i}=1 / \sqrt{r_{h, i}^{2}+x_{h, i}^{2}}$ - the admittance of the network node; $g_{h, i}=r_{h, i} / \sqrt{r_{h, i}^{2}+x_{h, i}^{2}}$ - the conductance

of network node, $b_{h, i}=x_{h, i} / \sqrt{r_{h, i}^{2}+x_{h, i}^{2}}-$ the susceptance of network node.

To detect the nodes and harmonics where resonant modes can occur, the author analyzes the values $K_{U(h)}$ and detects the nodes where standard values $K_{L}$ are exceeded. The large current is an indicator of the resonance in the node at the $h$-th harmonic. If there is a resonant mode in the node at some harmonic then the susceptance of the node at this harmonic is equal to zero and the sign of susceptance changes. The susceptance sign at series resonance is changed from negative to the positive one, and vice versa at parallel resonance. When the mode is close to resonant, the values of conductance and admittance are numerically close while the susceptance value is generally one order less than those of conductance and admittance ones. If the values $K_{U(h)}$ do not exceed limit values [14], one should conduct the analysis of admittances for detecting the nodes where resonance is possible. Then the analysis of susceptance of the presence of a sign change (from plus to minus or from minus to plus) should be performed. A sign change in a node at different harmonics can occur several times, which indicates resonance modes in the network at several harmonics at the same time. The mode close to the resonant one can be detected by the small values of susceptance comparing with admittance and conductance.

As a rule, the calculation of the network mode is made for the 3, 5, 7, 9, 11, 13, 17, 19, 23, 25-th harmonics. The values of coefficients $K_{U(h)}$ at these harmonics most frequently exceed limit values [14] in the network nodes. If, as a result of the calculation, it was found that the susceptance changes sign when the harmonics change as described above, then it is necessary to determine the interval of harmonics in which resonance is present and to clarify the harmonic. To clarify the resonant harmonic, it is necessary to calculate the frequency response of the susceptance with a certain step and depict it in a graph. After analyzing the frequency response, the harmonic interval should be reduced, and the frequency response should be calculated in smaller step. From the obtained frequency response of the susceptance the resonance harmonic should be determined again. In most cases such harmonics is not an integer number (interharmonic). Resonant circuits are formed on interharmonics. If necessary, the calculation is carried out again for a smaller step until the numbers of the resonant harmonics are established. After obtaining resonant harmonics one should assess the danger of resonance occurrence on this harmonics in the network. Resonance is dangerous if there is a current in the network with the number of the calculated harmonic. In order to get a complete picture of the resonance capabilities of the network at various harmonics, it is necessary to analyze the possible resonances arising from various changes in the network, which can cause a shift in the resonance harmonics, for 
example, turning on and off power lines and capacitors, changing load values, etc.

\section{Examples of Resonance Modes on Harmonic Frequencies in Electric Networks of Eastern Siberia}

An analysis of several resonant modes in electric networks of Eastern Siberia was performed using the developed methodological approach and HARMONICS software. The results of calculations and analysis are presented below.

\section{A. Resonance Modes at the $220 \mathrm{kV}$ Pokosnoe Substation}

As an example the analysis of one of the network nodes in Eastern Siberia with the number 2401 is performed. The $220 \mathrm{kV}$ Pokosnoe substation is located in this node. Table I provides the calculated parameters of the node and of the mode for 10 harmonics.

Table I. - Parameters of the node and mode

\begin{tabular}{|c|c|c|c|c|c|c|}
\hline$h$ & $\begin{array}{c}K_{U(h)} \\
(\%)\end{array}$ & $\begin{array}{c}K_{L} \\
(\%)\end{array}$ & $\begin{array}{c}y_{h} \\
(\mathrm{~S})\end{array}$ & $\begin{array}{c}g_{h} \\
(\mathrm{~S})\end{array}$ & $\begin{array}{c}b_{h} \\
(\mathrm{~S})\end{array}$ & $\begin{array}{c}I_{h} \\
(\mathrm{~A})\end{array}$ \\
\hline 3 & 0.98 & 1.50 & 0.0066 & 0.0009 & -0.0065 & 21.31 \\
\hline 5 & 1.15 & 1.50 & 0.0028 & 0.0005 & -0.0028 & 10.68 \\
\hline 7 & 1.67 & 1.00 & 0.0020 & 0.0015 & -0.0013 & 7.29 \\
\hline 9 & 0.69 & 0.40 & 0.0045 & 0.0037 & -0.0026 & 2.78 \\
\hline 11 & 1.22 & 1.00 & 0.0027 & 0.0024 & 0.0013 & 7,38 \\
\hline 13 & 1.72 & 0.70 & 0.0036 & 0.0036 & -0.0001 & 9.68 \\
\hline 17 & 0.18 & 0.50 & 0.0126 & 0.0085 & 0.0092 & 2.47 \\
\hline 19 & 0.22 & 0.40 & 0.0235 & 0.0234 & -0.0008 & 4.60 \\
\hline 23 & 0,23 & 0.40 & 0.0142 & 0.0112 & -0.0087 & 2.91 \\
\hline 25 & 0.13 & 0.40 & 0.0069 & 0.0057 & -0.0038 & 0.78 \\
\hline
\end{tabular}

The table analysis shows that the coefficients $K_{U(h)}$ exceed the standard values [14] on 7, 9, 11 and 13-th harmonics. Conductance and susceptance of the 7-th harmonic have alike values that is why it is possible that $K_{U(h)}$ exceeds the standard value $K_{L}$ due to a higher value of the 7-th current harmonic. Susceptance has a negative sign at the 9-th harmonic and a positive sign at the 11-th harmonic, which indicates a series resonance in the harmonic interval from 9 to 11 -th. Admittance and conductance of the 11-th harmonic have alike values while susceptance is two times less comparing with the admittance and conductance but the value is of the same order as the admittance has, i.e. resonance is possible. Admittance and conductance of the 13-th harmonic are virtually equal. Susceptance is one order less than the admittance and conductance. The susceptance changes the sign from positive to the negative one. This implies that on the 13-th harmonic in the node the mode is close to the resonant mode. The change in the susceptance sign from negative to the positive one at the interval of the 13 - 17-th harmonics, which means there is series resonance. Between the 17 and 19-th harmonics one can observe the change of the sign from positive to negative which shows parallel resonance. Therefore, in the interval of the 9 19-th harmonics one can observe resonant circuit in the network in relation to the analyzed node.

To clarify the resonance harmonics one should calculate the frequency responses of the admittance, conductance and susceptance for the analyzed node with the help of HARMONICS software. Figure 1 shows these frequency responses for the node 2401 in the interval of harmonics from 3-rd to 25-th. The figure shows that in the node on the harmonic from 9-th to 19-th there are four resonances: two series and two parallel. Resonances alternate. To determine the resonant harmonic, it is possible to calculate the frequency response in smaller step, for example, $0.02 \%$ of the order of the lower harmonic.

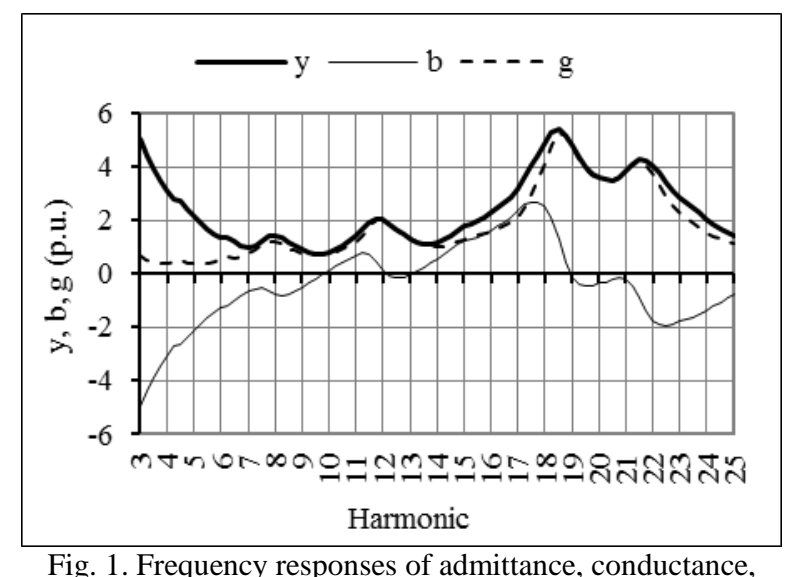

Fig. 1. Frequency responses of admittance, conductance, susceptance on the 3-25-th harmonics.

Figure 2 shows the frequency responses in the interval of the 9-13-th harmonics. The analysis of frequency responses demonstrates that the first resonance occurs approximately on the harmonic 9.8, the second occurs on the harmonic 12.2, the third resonance is observed on the 13-th harmonic.

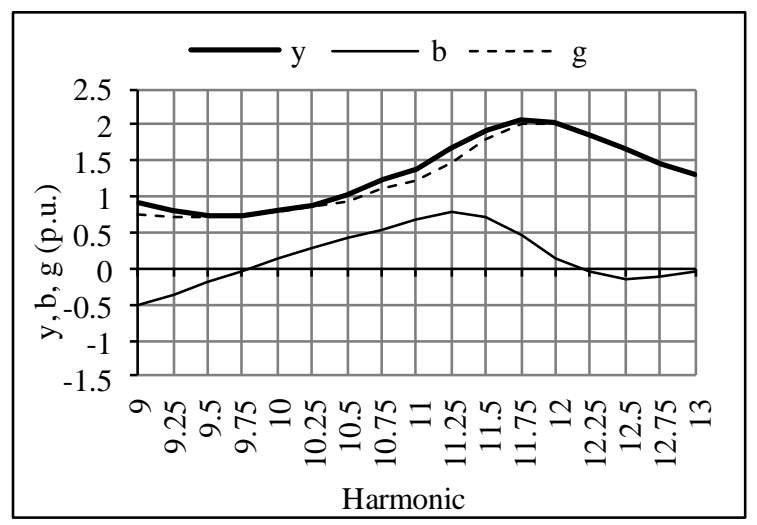

Fig. 2. Frequency responses of admittance, conductance, susceptance on the 9-13-th harmonics.

One could consider the switch off of power lines, connected to the node 2401, as the network changes which can cause the shift of resonant points in relation to the harmonic number in the analyzed node. Two $220 \mathrm{kV}$ lines are connected to the node 2401: 2401-2280 and 2401-2402. We calculate the frequency responses of susceptance of the node 2401 , turning off one of the lines. Figures 3 provide the frequency responses of the node susceptance for three cases: both lines are turned 
on; the line 2401-2280 is turned on and the line 2401-2402 is turned off; the line 2401-2280 is turned off and the line 2401-2402 is turned on. Analysis shows that network configuration influences the occurrence of resonances. When the line 2401-2402 is turned off, the resonances occur close to the $6,8,9,12,14$-th and 21-st harmonics. When the line 2401-2280 is turned off, resonances in the network in relation to the node 2401 occur approximately on the harmonics 12.5 and 18.6.

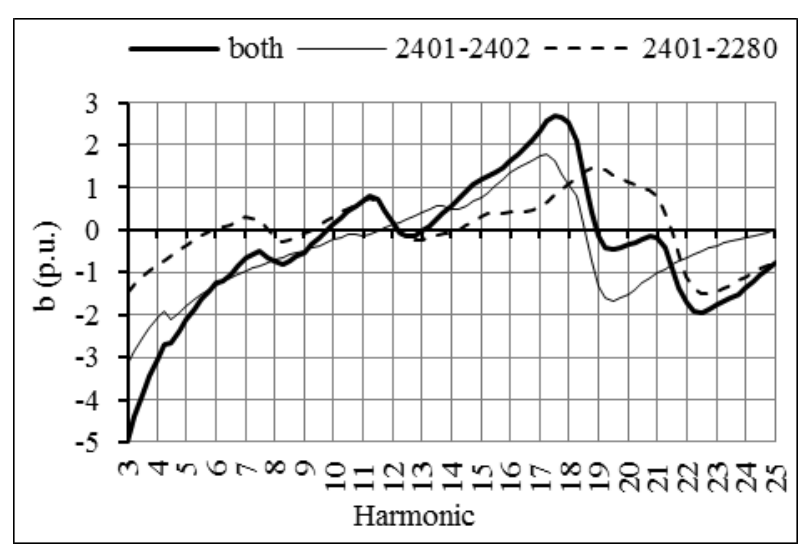

Fig. 3. Frequency response of susceptance for node 2401.

\section{B. Analysis of Resonance Modes in the $110 \mathrm{kV}$ Network Node}

Figure 4 illustrates the frequency responses of input admittance, susceptance and conductance of the $110 \mathrm{kV}$ network node. Taksimo railway substation is connected to this node. The substation gains power through the autotransformer from the $220 \mathrm{kV} 1000 \mathrm{~km}$ long electric network that powers other substations of the railway.

To analyze the resonant modes in a $220 \mathrm{kV}$ network, we used the scheme network of East Siberia, which has 542 nodes with voltages from 10 to $500 \mathrm{kV}$. The nodes include the following: 27 nodes with generators, 300 nodes with active-inductive and asynchronous load, 81 nodes with large non-linear loads (including railway substations and aluminum plant), 98 nodes with shunt reactors and capacitors, 6 nodes with equivalent circuits of electric network containing non-linear loads. The scheme has 682 connecting link, including power lines, transformers, autotransformers and series compensators.

In the node where Taksimo substation is connected to the network the harmonic factors $K_{U(h)}$ were measured. They were used to model the modes of the electric network. For example, the measured value of $K_{U(5)}$ turned out to be equal $2.57 \%$. After modeling the mode of the 5-th harmonic, the calculated $K_{U(5)}$ value in the node turned out to be close to the measured value and amounted to $2.30 \%$. The calculations showed that at the 5-th harmonic in the connection node of the Taksimo railway substation there is the mode close to the resonance one. The susceptance curve (Figure 4) suggests that conductance turns positive from negative and thereby illustrating series resonance circuits on harmonics 4.5, 6.6, and 8.5. Susceptance turns negative from positive thereby illustrating parallel resonance modes on harmonics 4.9, 7.1 and 10.9. The mentioned harmonics are interharmonics. If currents of the mentioned harmonics are not present in the electric network, then resonance circuits on those harmonics do not constitute any danger.

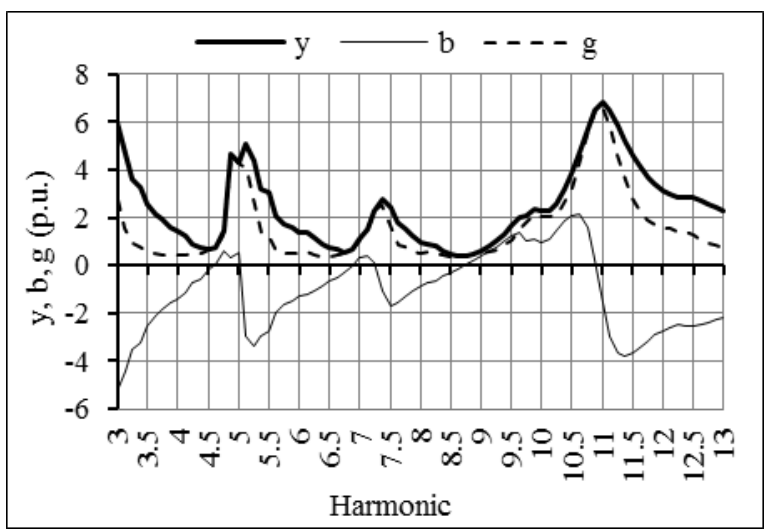

Fig. 4. Frequency responses on the 3-13-th harmonics at the Taksimo railway substation.

\section{Analysis of Resonance Modes in the $220 \mathrm{kV}$ Network}

Figure 5 illustrates values calculated with the use of HARMONICS software: current, susceptance, admittance and harmonic factor for the 5-th harmonic in nodes of the $220 \mathrm{kV}$ networks.

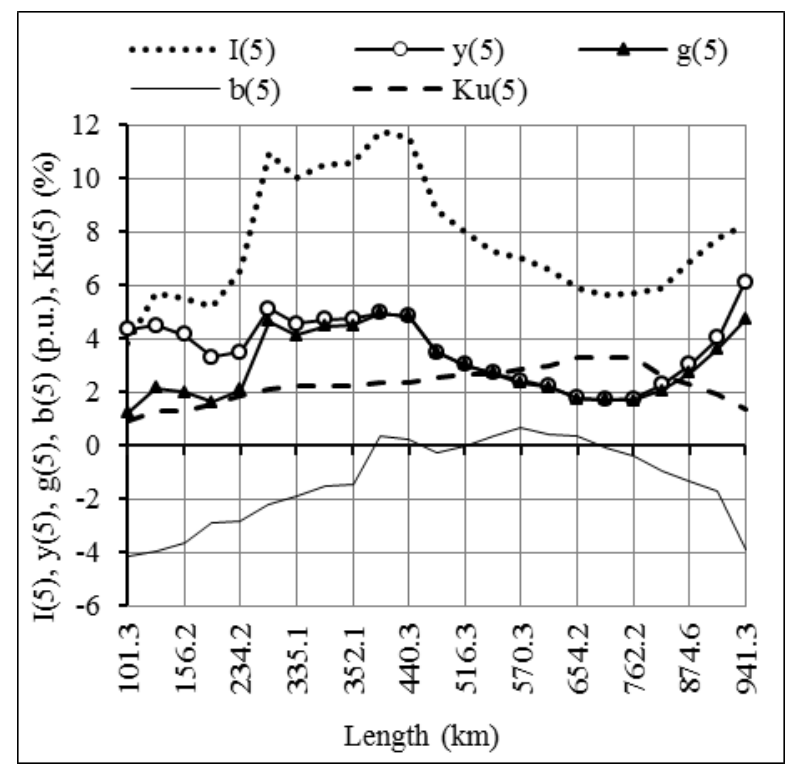

Fig. 5. Parameters of nodes and mode of $220 \mathrm{kV}$ network on the 5-th harmonic

This network powers 23 substations from Slyudyanka $(\sim 101.3 \mathrm{~km}$ in Fig. 5) to Chita ( 941.3 km in Fig. 5). Those two substations are some $840 \mathrm{~km}$ far from each other. The curves in the figure show that resonance modes occur on the interval from 400 to $700 \mathrm{~km}$. Susceptance in this area changes its sign four times. In the analyzed area, two series resonance circuits and two parallel resonance circuits occur on the 5-th harmonic. As susceptance falls, admittance also decreases and becomes equal to conductance. Despite the 5-th current harmonic decreases, $K_{U(5)}$ increases, this may be due to the resonance mode in the electric network. Measurements performed on substations confirmed high $K_{U(5)}$. At 
Novoilyinsky substation $\left(\sim 516.3 \mathrm{~km}\right.$ in Fig. 5) $K_{U(5)}$ totaled $2.07 \%$. Value $K_{U(5)}$ amounted to $2.27 \%$ at Kizha substation $(\sim 544.3 \mathrm{~km}$ in Fig. 5). Both values exceed the limit value $1.5 \%$ [14].

\section{Analysis of the Resonance Modes that Occurs when the Capacitor Bank is Turned on}

The capacitor bank is located at the substation Tataurovo, which belongs to the power supply organization. The capacitor bank has a capacity of 50 Mvar. It is turned on by the operator command. The capacitor bank is designed to maintain the voltage at the fundamental frequency that meets regulatory requirements.

Figure 6 illustrates the $K_{U(h)}$ value measured in phases B and $\mathrm{C}$ at the Tataurovo railway substation before and after turning on the capacitor bank. The capacitor bank was turned on the 19-th minute. The curves suggest that values $K_{U(3)}$ and $K_{U(5)}$ increased after turning on the capacitor bank.

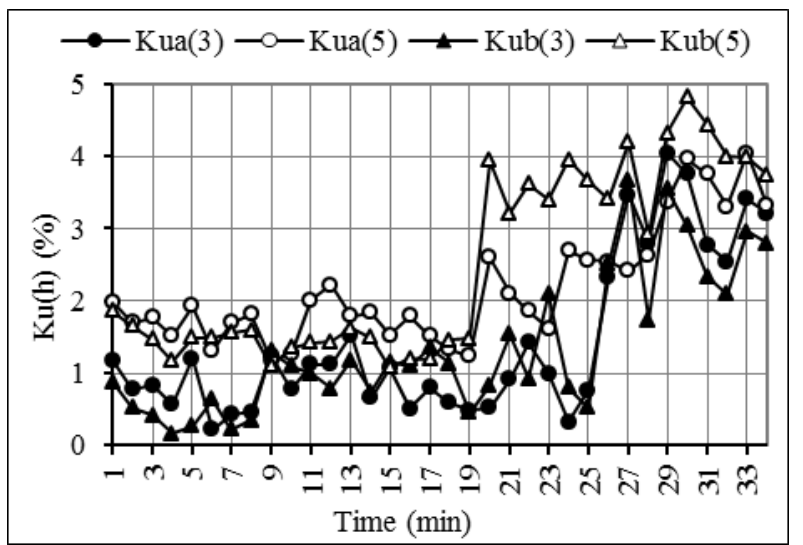

Fig. 6. $K_{U(h)}$ at Tataurovo railway substation.

HARMONICS software helped analyze how the capacitor bank affects the parameters of the network and mode of Tataurovo railway substation. Calculation results for phase A are given in Table II.

Table II. - Parameters of the electric network and mode in the node connection of Tataurovo railway substation

\begin{tabular}{|l|c|c|}
\hline \multirow{2}{*}{ Parameter } & \multicolumn{2}{|c|}{ Harmonic } \\
\cline { 2 - 3 } & 3 & 5 \\
\hline $\mathrm{I}(\mathrm{h})(\mathrm{A})$ & 7.8143 & 14.9198 \\
\hline $\mathrm{g}(\mathrm{h}) \mathrm{CB}(\mathrm{S})$ & 0.0 & 0.0 \\
\hline $\mathrm{g}(\mathrm{h}) \mathrm{NW}(\mathrm{S})$ & 0.0046 & 0.0092 \\
\hline $\mathrm{g}(\mathrm{h}) \mathrm{CB}+\mathrm{NW}(\mathrm{S})$ & 0.0046 & 0.0092 \\
\hline $\mathrm{b}(\mathrm{h}) \mathrm{CB}(\mathrm{S})$ & 0.0076 & 0.0127 \\
\hline $\mathrm{b}(\mathrm{h}) \mathrm{NW}(\mathrm{S})$ & -0.0116 & -0.0125 \\
\hline $\mathrm{b}(\mathrm{h}) \mathrm{CB}+\mathrm{NW}(\mathrm{S})$ & -0.0039 & 0.0003 \\
\hline $\mathrm{y}(\mathrm{h}) \mathrm{CB}+\mathrm{NW}(\mathrm{S})$ & 0.0060 & 0.0092 \\
\hline$K_{U(h)}(\%)($ without CB) & 1.14 & 1.75 \\
\hline$K_{U(h)}(\%)($ with CB) & 2.36 & 2.96 \\
\hline$K_{L}(\%)$ & 1.5 & 1.5 \\
\hline
\end{tabular}

The notations used in the table are: $\mathrm{I}(\mathrm{h})$ - the current of the $h$-th harmonic in the electric network node; $\mathrm{g}(\mathrm{h}) \mathrm{CB}$ the conductance of the capacitor bank; $g(h) N W$ - the input conductance of the electric network node; $\mathrm{g}(\mathrm{h}) \mathrm{CB}+\mathrm{NW}$ - the sum of conductance of the capacitor bank and input conductance of the electric network node; $b(h) C B$ - the susceptance of the capacitor bank; b(h)NW - the input susceptance of the electric network node; $\mathrm{b}(\mathrm{h}) \mathrm{CB}+\mathrm{NW}$ - the sum of susceptance of the capacitor bank and input susceptance of the electric node; $\mathrm{y}(\mathrm{h}) \mathrm{CB}+\mathrm{NW}$ - the admittance of the capacitor bank and electric network node.

The table shows the calculated of $K_{U(h)}$ values in the network node before and after turning on the capacitor bank; $K_{L}$ is the standard value of the $h$-th harmonic factor. As seen from the table, before the capacitor bank turning on, input susceptance of the network node on the 3-rd and 5-th harmonics was inductive (negative). After the capacitor bank turning on, susceptance of the 5-th harmonic turned positive, which suggests the occurrence of the resonance mode between the 3-rd and 5-th harmonics. As susceptance turned positive from negative, a series resonance occurred. The values of $K_{U(3)}$ and $K_{U(5)}$ increased and significantly exceeded the $K_{L}$.

\section{Conclusion}

Resonance modes in electrical networks occur randomly by various changes in the network configuration and load powers, as well as turning on capacitor banks and and passive harmonic filters.

Resonant circuits and modes in high voltage electrical networks can be determined and analyzed using special software. Resonance modes are one of the reasons for the high values of voltage and current harmonics. This knowledge is very important due to the widespread availability of digital equipment that is sensitive to low quality voltage.

Special software can be used in the operation of electric networks for predicting resonance modes at various harmonics during power quality control.

\section{Acknowledgement}

The research is conducted in the framework of the research projects №AAAA-A17-117030310432-9 and №AAAA-A17-117030310438-1 of the program of fundamental research of Siberia Branch of the Russian Academy of Sciences III.17.4.

\section{References}

[1] D.K. Geddey, D.C. Smith, P.O.Wright, P.M. Hart, G.E. Littler, "Control of harmonic distortion on Australian Electricity supply networks". CIGRE, 1986, Paper 36-10/ In the book "The impact of high voltage electrical installations on the environment". Moscow, 
Energoatomizdat, 1988, pp. 51-59.

[2] K. Hlava, I. Kabrhel, J. Smid, M. Blasko,"Experimental investigation of harmonic impedances and other electrical parameters of high voltage network". CIGRE, 1986, Paper 36-04 / In the book "The impact of high voltage electrical installations on the environment". Moscow,

Energoatomizdat, 1988, pp. 59-64.

[3] Tutorial harmonics modeling and simulation. IEEE power engineering society. IEEE Catalog number: 98TR125-0.

[4] T. Vinnal, T. Sakkos, K. Janson, M. Jarkovoi, "Resonances in LV industrial networks when using shunt capacitors for power factor correction", Proceedings of 23th International Conference on Electricity Distribution - CIRED 2015, Lyon, 15-18 June 2015.

[5] J. Meyer, R. Stiegler, P. Schegner, I. Röder, A. Belger, "Harmoic resonances in residential low voltage networks caused by consumer electronics", Proceedings of 24th International Conference on Electricity Distribution CIRED 2017, Glasgow, 12-15 June 2017.

[6] L. Eggenschwiler, O. Galland, D. Chollet, F. Decorvet, D. Roggo, P. Favre-Perrod, "Frequency scans and resonance mode analysis for resonance problems identification in power networks in presence of harmonic pollution", Proceedings of 24th International Conference on Electricity Distribution - CIRED 2017, Glasgow, 12-15 June 2017.

[7] B. Mohamadi Kalesar, J. Behkesh Noshahr, "Capacitor bank behavior of cement factory in presence of supraharmonics resulted from switching full power frequency converter of generator (PMSG)", Proceedings of 24th International Conference on Electricity Distribution - CIRED 2017, Glasgow, 12-15 June 2017.

[8] A. Bottenberg, C. Debruyne, B. Peterson, J. Rens, J. Knockaert, J. Desmet, "Network resonance detection using harmonic active power", Proceedings of 18th International Conference on Harmonics and Quality of Power (ICHQP), May 13-16, 2018, Ljubljana, Slovenia.

[9] T. Busatto, V. Ravindran, A. Larsson, M. H. J. Bollen, "Estimation of the consumer electronics capacitance for harmonic resonance studies by a non-invasive measurement method", Proceedings of 18th International Conference on
Harmonics and Quality of Power (ICHQP), May 13-16, 2018, Ljubljana, Slovenia.

[10] RD 153-34.0-15.501-00. Guidelines for monitoring and analyzing the power quality in public power supply systems. Analysis of the power quality. Moscow, 2002.

[11] Unified technical policy in the power grid complex of "Rosseti". Project 2019. Moscow.

[12] L.A. Neiman, K.S. Demirchan, "Theoretical foundations of electrical engineering": In 2 Vol. Textbook for universities. Volume 1. - 3rd ed., revised. and add. - L.: Energoatomizdat. Leningr. department, 1981.

[13] G.J. Wakileh, "Power system harmonics: fundamentals, analysis and filter design". - Berlin; Heidelberg; New York; Barcelona; Hong Kong; Paris: Springer, 2001.

[14] GOST 32144-2013. Electric energy. Electromagnetic compatibility of technical equipment. Power quality requirements in public electricity supply systems. Moscow, Standartinform, 2014.

[15] J. Arrilaga, D. Bradley, P. Boger, "Harmonics in electrical systems", Energoatomizdat, 1990

[16] I.V. Zhezhelenko, "Higher harmonics in power supply systems of industrial enterprises". M.: Energoatomizdat, 2010.

[17] J.M.A. Bhattacharyya, W.L. Myrzik, W.L. Kling, "Consequences of poor power quality - An overview". International Universities Power Engineering Conference, Brighton (UR), 4th to 6th September, 2007.

[18] L.I. Kovernikova, "HARMONICS software for analysis and normalization of harmonic modes on HV networks", Proceedings of the International Scientific and Practical Conference, Moscow, 23-25 November 2016, https://icpqm.org/media/medialibrary/2017/06/PQM2016_Proceedings.pdf

[19] L.I. Kovernikova, Analysis of probabilistic properties of harmonic currents of loads connected to the high voltage networks", Proceedings of the International Conference on Renewable Energies and Power Quality (ICREPQ'14), La Coruña (Spain), 23th to 27th March, 2015.

[20] L.A. Zhukov, I.P. Stratan, "Steady-state modes of complex electrical networks and power systems: calculation methods". Energiya, 1979. 